\title{
Ocorrência do fungo entomopatogênico Isaria javanica (Frieder. \& Bally) Samson \& Hywell-Jones (Fungi, Sordariomycetes) em lagartas de Lonomia obliqua Walker (Lepidoptera, Saturniidae, Hemileucinae)
}

\author{
Alexandre Specht ${ }^{1,2}$, João Lúcio Azevedo², Elza Áurea de Luna Alves Lima³, Juliano Tomazzoni Boldo², \\ Mayra Kassawara Martins ${ }^{4}$, Lisete Maria Lorini ${ }^{5} \&$ Neiva Monteiro Barros ${ }^{2}$
}

${ }^{1}$ Centro de Ciências Exatas, da Natureza e de Tecnologia, Universidade de Caxias do Sul, Campus Universitário da Região dos Vinhedos. Caixa Postal 32, 95700-000 Bento Gonçalves-RS, Brasil. aspecht@ucs.br.

${ }^{2}$ Instituto de Biotecnologia, Centro de Ciências Agrárias e Biológicas, Universidade de Caxias do Sul, Cidade Universitária. Caixa Postal 1352, 95070-560 Caxias do Sul-RS, Brasil.

${ }^{3}$ Departamento de Micologia, Centro de Ciências Biológicas, Universidade Federal de Pernanbuco, 50670-901, Recife-PE, Brasil.

${ }^{4}$ Centro de Biotecnologia da Amazônia, 69075-351 Manaus-AM, Brasil.

${ }^{5}$ Instituto de Ciências Biológicas, Universidade de Passo Fundo, Caixa Postal 611, 99001-970 Passo Fundo-RS, Brasil.

\begin{abstract}
Occurrence of the entomopathogenic fungus Isaria javanica (Frieder. \& Bally) Samson \& Hywell-Jones (Fungi, Sordariomycetes) infecting Lonomia obliqua Walker (Lepidoptera, Saturniidae, Hemileucinae) caterpillars. It is recorded for the first time the occurrence of the entomopathogenic fungus Isaria javanica (Frieder. \& Bally) Samson \& Hywell-Jones (Fungi: Sordariomycetes) infecting Lonomia obliqua Walker (Lepidoptera: Saturniidae: Hemileucinae) caterpillars. This fungus was isolated from L. obliqua individuals collected from Platanus acerifolia (Aiton) WildPlatanaceae in Rio Grande do Sul state, Brazil. After isolation, purification and characterization, fungal conidia were inoculated on healthy L. obliqua caterpillars and from dead caterpillars the fungal isolates were again obtained. New isolates and the original isolate did not differ when compared by morphological and molecular tests.
\end{abstract}

KEYWORDS. Biological control; natural enemies; urticant caterpillars.

RESUMO. Ocorrência do fungo entomopatogênico Isaria javanica (Frieder. \& Bally) Samson \& Hywell-Jones (Fungi, Sordariomycetes) atacando lagartas de Lonomia obliqua Walker (Lepidoptera, Saturniidae, Hemileucinae). Este fungo foi isolado pela primeira vez de lagartas de L. obliqua de uma agregação em plátano (Platanus acerifolia (Aiton) Wild Platanaceae), em Bento Gonçalves, RS, Brasil. Após isolamento, purificação e caracterização, realizou-se um teste de patogenicidade com lagartas sadias de L. obliqua para corroborar, sua infectividade pelo postulado de Koch. Constatouse correspondência morfológica e molecular entre o inóculo e o reisolado, comprovando sua patogenicidade a $L$. obliqua.

PALAVRAS-CHAVE. Controle biológico; inimigos naturais; lagartas urticantes.

Os representantes do gênero Lonomia Walker, 1855 destacamse entre os lepidópteros de importância médica devido às suas lagartas serem capazes de produzir e inocular substâncias de ação urticante e de efeito hemorrágico em humanos; são responsáveis por acidentes graves em toda a região Neotropical com o registro de diversos óbitos (Lemaire 2002).

As 11 espécies do gênero têm distribuição Neotropical, sendo que a distribuição de Lonomia obliqua Walker, 1855 acompanha o bioma da Mata Atlântica desde a Bahia até o Rio Grande do Sul, havendo registros também no Uruguai (Lemaire 2002). Sua importância aumentou muito nas últimas duas décadas devido a níveis populacionais elevados, causando muitos acidentes principalmente nos estados do Rio Grande do Sul e de Santa Catarina (Lorini 1999). Alguns estudos com esta espécie relacionam as possíveis causas do seu aumento populacional, sendo as principais, alterações ambientais e diminuição de seus inimigos naturais.
A presente nota relata a ocorrência do fungo Isaria javanica (Frieder \& Bally) Samson \& Hywell-Jones (Fungi, Sordariomycetes) pertencente família Cordycipitaceae e Divisão Hypocreales. O mesmo foi isolado de lagartas de $L$. obliqua coletadas na localidade Linha Brasil, Distrito de Pinto Bandeira, Bento Gonçalves, Rio Grande do Sul, Brasil. As mesmas foram provenientes de uma agregação de 14 lagartas encontradas no dia 30 de maio de 2003, no tronco de um plátano [Platanus acerifolia (Aiton) Wild. - Platanaceae]. As larvas foram mantidas no Laboratório de Biologia do Campus Universitário da Região dos Vinhedos, Universidade de Caxias do Sul, em gaiolas teladas de $40 \times 50 \mathrm{~cm}$ de base e $80 \mathrm{~cm}$ de altura, mantidas a $25 \pm 1^{\circ} \mathrm{C}, 70 \pm 10 \%$ UR e 14 horas de fotofase, alimentadas com folhas de $P$. acerifolia. Dois exemplares deixaram de se alimentar, abandonaram a agregação com sinais de diminuição dos movimentos e paralisia progressiva, indicando o possível ataque de microrganismos. Tais larvas 
foram mantidas individualmente em placas de Petri, cujo fundo foi forrado com papel filtro, umedecido com água destilada no Laboratório de Controle de Pragas do Instituto de Biotecnologia da Universidade de Caxias do Sul, Rio Grande do Sul. Após a morte os insetos foram transferidos para câmara úmida até o completo desenvolvimento do entomopatógeno. A seguir, foi feita a desinfeção do corpo dos insetos, mergulhando-os, com agitação durante três minutos, em uma solução de hipoclorito de sódio (2,5\% de cloro ativo, v/v), seguindo-se três lavagens sucessivas em solução salina $(0,87 \%)$, para eliminação do excesso de cloro. As lagartas foram finalmente transferidas para placas de Petri contendo meio de Batata Dextrose Agar (BDA), acrescido de Ampicilina, incubadas em B.O.D. a $26^{\circ}$ C. A purificação do fungo foi feita a partir das colônias formadas na superfície do meio a fim de se obter culturas puras as quais foram transferidas para tubos de ensaio contendo meio BDA.

A classificação foi feita com base na morfologia dos conídos, fiálides e hifas utilizando chave dicotômica (Humber 1998) e análise molecular com sequenciamentos feitos no Laboratório de genômica da USP.

A atualização taxonômica foi realizada com base em Hibbett et al. (2007) e Humber (2007). Os isolados foram incluídos na coleção de fungos do Departamento de Micologia da Universidade Federal de Pernambuco, Recife, PE, sob números URM 4993 e URM 4995, tendo sido classificados como $I$. javanica.

Para confirmar o potencial entomopatogênico do fungo, inoculou-se $9 \mathrm{~mL}$ de uma suspensão de 5,2 x $10^{7}$ conídios por mL do isolado URM 4993 em uma agregação de 40 lagartas sadias de L. obliqua, de quarto ínstar, proveniente de Água Santa, Rio Grande do Sul. Todas as lagartas morreram até o sétimo dia após a inoculação sendo que o patógeno foi recuperado a partir de três lagartas mortas. Tanto o sequenciamento do fungo isolado (duas repetições) das lagartas coletadas na natureza, quanto o do re-isolado (duas repetições) após bioensaio comprovou se tratar da mesma espécie, confirmando a capacidade de $I$. javanica em infectar larvas de L. obliqua. A sequência foi depositada no GenBank sob o código de acesso FJ750877.

Os fungos entomopatogênicos são eficientes agentes de controle biológico. Dentre eles, espécies do gênero Isaria Pers., 1797 foram encontradas atacando insetos de diversas ordens, bem como nematódeos. No Brasil, representantes deste gênero, causador da chamada "muscardine amarela dos insetos" já foram isolados de Stenoma decora, Zeller 1854 (Lepidoptera, Elachistidae), Eupseudosoma spp. (Lepidoptera, Arctiidae) e Lagria vilosa Fabricius, 1783 (Coleoptera: Lagriidae) tendo sido também encontrado causando epizootias em lagartas de Brassolis sp. (Lepidoptera, Nymphalidae) (Alves 1998).

Das espécies deste gênero, no Brasil, destacam-se $I$. farinosa, I. tenuipe (Peck) Samson, I. cicadidae (Miquel.)
Samson, I. fumosorosea (Wize) Brown \& Smith e I. amoenorosea (Hennings) Samson (Alves 1998) removidas do gênero Paecilomyces Bainier (Luangsa-Ard et al. 2005; Hibbett et al. 2007). Recentemente, I. javanica foi encontrada pela primeira vez na América do Sul (Argentina) por Scorsetti et al. (2008) infectando Trialeurodes vaporariorum Westwood (Hemiptera, Aleyrodidae).

Esta nota relata pela primeira vez, a ocorrência de um fungo entomopatogênico atacando lagartas de L. obliqua e, é também o primeiro relato de I. javanica isolado de um representante de Saturniidae. A necessidade de minimizar os acidentes causados pelas lagartas de L. obliqua tem levado a busca de alternativas para o controle deste inseto sendo a utilização de fungos entomopatogênicos, como I. javanica, uma alternativa promissora.

Agradecimentos. À Fundação de Amparo a Pesquisa do Rio Grande do Sul (FAPERGS), pela concessão do auxílio PROADE 2 02/1739.6 e $\mathrm{CNPq}$ pela concessão de bolsa de iniciação cientifica. Aos revisores pela extensiva revisão e contribuição.

\section{REFERÊNCIAS}

Alves, S. B. 1998. Fungos entomopatogênicos, p. 289-381. In: S. B. Alves (ed.) Controle Microbiano de Insetos. Piracicaba, FEALQ, $1163 \mathrm{p}$.

Hibbett, D. S.; M. Binder; J. F. Bischoff; M. Blackwell; P. F. Cannon; O E. Eriksson; S. Huhndorf; T. James; P. M. Kirk; R. Lücking; T. Lumbsch; F. Lutzoni; P. B. Matheny; D. J. Mclaughlin; M. J. Powell; S. Redhead; C. L. Schoch; J. W. Spatafora; J. A. Stalpers; R. Vilgalys; M. C. Aime; A. Aptroot; R. Bauer; D. Begerow; G. L. Benny; L. A Castlebury; P. W. Crous; Y.-C. Dai; W. Gams; D. M. Geiser; G. W. Griffith; C. Gueidan; D. L. Hawksworth; G. Hestmark; K. Hosaka; R. A. Humber; K. Hyde; J. E. Ironside; U. Kõljalg; C. P. Kurtzman; K.-H. Larsson; R. Lichtwardt; J. Longcore; J. Mi_dlikowska; A. Miller; J.-M. Moncalvo; S. Mozley-Standridge; F. Oberwinkler; E. Parmasto; V. Reeb; J. D. Rogers; C. Roux; L. Ryvarden; J. P. Sampaio; A. Schüßler; J. Sugiyama; R. G. Thorn; L. Tibell; W. A. Untereiner; C. Walker; Z. Wang; A. Weir; M. Weiß; M. M. White; K. Winka; Y.-J. Yao \& N. Zhang. 2007. A higher-level phylogenetic classification of the Fungi. Mycological Research 111: 509547.

Humber, R. A. 1998. Entomopathogenic fungal identification. APS/ ESA workshop. http://www.ppru.cornell.edu/mycology/ Insect_mycology.html.

Humber, R. A. 2007. Recent phylogenetically based reclassifications of fungal pathogens of invertebrates. New York: ARSEF, 5p. Disponível em: http://www.sipweb.org/fungi/ humber.pdf.

Lemaire, C. 2002. The Saturniidae of America - Hemileucinae Keltern, Goecke \& Evers, 3 v., 1388 p.

Lorini, L. M. 1999. A taturana - Aspectos biológicos e morfológicos da Lonomia obliqua. Passo Fundo, EDIUPF, 67 p.

Luangsa-Ard, J. J.; N. L. Hywel-Jones; L. Manoch \& R. A. Samson. 2005. On the relationships of Paecilomyces sect. Isarioidea species. Mycological Research. 109: 581-589.

Scorsetti, A. C.; R. A. Humber; C. De Gregório \& C. C. L. Lastra. 2008 New records of entomopathogenic fungi infecting Bemisia tabaci and Trialeurodes vaporariorum pests of horticultural crops in Argentina. BioControl 53: 787-796.

Recebido em 05/11/2007; aceito em 26/02/2009 